Gazi University
Journal of Science
http://dergipark.gov.tr/gujs

\title{
Reliable Iterative Methods for Solving the Falkner-Skan Equation
}

\author{
Majeed A. AL-JAWARY*(D), Montaser I. ADWAN (D) \\ Department of Mathematics, College of Education for Pure Sciences (Ibn AL-Haitham) / University of Baghdad, Iraq, Baghdad
}

\author{
Highlights \\ -Reliable iterative methods to solve the Falkner-Skan problem to get new approximate solutions. \\ -We compare the obtained numerical results with the Runge-Kutta (RK4) and Euler methods. \\ -The fixed point theorem is presented to test the convergence of the suggested techniques. \\ -The results of the remaining maximum error values showing that the techniques are reliable and effective.
}

\begin{tabular}{l} 
Article Info \\
Received: $06 / 09 / 2018$ \\
Accepted: $04 / 10 / 2019$ \\
Keywords \\
\hline Falkner-Skan equation \\
Iterative methods \\
Numerically solution
\end{tabular}

\begin{abstract}
In this work, we suggest reliable iterative methods to solve the Falkner-Skan problem to obtain new approximate solutions. The suggested methods are Tamimi-Ansari method (TAM), Daftardair-Jafari method (DJM) and Banach countraction method (BCM). We compare the obtained numerical results with other numerical methods like the Runge-Kutta (RK4) and Euler methods. The fixed point theorm is presented to test the convergence of the suggested methods. Moreover, the results of the remaining maximum error values showing that the suggested methods are reliable and effective. The Software used in our calculations for this work is Mathematica ${ }^{\circledR}$ 10 .
\end{abstract}

\section{INTRODUCTION}

The Falkner-Skan problem was first studied in 1931 by Falkner and Skan [1]. The Falkner-Skan equation has many important applications in industrial processes, such as drawing of plastic films, metal spinning, cooling of a metallic plate in a cooling bath, an aerodynamic extrusion of plastic sheets, metallic plates and others [2]. There are many methods used to solve the Falkner-Skan problem, like Adomian decomposition method (ADM) [2], Variational iteration method (VIM) [3,4], Optimal homotopy asymptotic method(OHAM) [5]. An iterative finite deference method(IFDM) [6], Homotopy Perturbation method (HPM) [7], Homotopy analysis method (HAM) [8], Runge-Kutta 4 (RK4) with shooting techniques [9] and collocation method [10]. In addition, there are many methods that give an approximate solution of the ordinary differential equations and partial equations such as variational methods (VIM)[11], Spline approximation method [12] and modified picard iteration method [13] and others.

A new iterative method was suggested by Daftardar-Gejji and Jafari (DJM) [14]. The Daftardar-Gejji method has been used to solve many nonlinear problems. Like application to partial differential equations [15], fractional boundary value problems with Dirichlet boundary conditions [16], Duffing equations [17], exact solutions of Laplace equation[18], Korteweg-de Vries equations [19], nonlinear ordinary differential equations (ODE) [20], and Volterra integro-differential equations [21].

Also, Temimi and Ansari (TAM) [22] have suggested a new semi- analytical iterative technique to solve the nonlinear equations. The Temimi and Ansari methods used to solve many differential problems, such as Duffing equation [23], solving chemistry problems [24], differential algebraic equations [25], nonlinear thin film flow equations [26], and Fokker-Planck's equations [27]. 
Moreover, another iterative method called the Banach Contraction Principle (BCP) is suggested by Daftardar-Gejji and Sachin Bhalekar [28]. The method has been used to solve various kinds of differential and integral equations [29].

The main goal of this paper is to implement the three iterative methods TAM, DJM and BCM to find approximate solutions for Falkner-Skan equation.

The work organized as follows: In section 2, the standard formulas of Falkner-Skan equation is provided. In section 3, shows the basic concepts of the reliable proposed methods are presented. In section 4, The Adomiani decompositions method(ADM). In section 5, convergence analysis of the suggested methods is given. In section 6, clarify and discuss the numerical simulation. Finally, in section 7 the conclusion is given.

\section{THE FORMULATION OF THE FALKNER-SKAN EQUATION}

The Falkner-Skan equation is classified as one of the nonlinear third-order ordinary differential equations. It has modeled a variety of important physical applications, such as insulating materials, applications of glass and polymer studies[9]. How to deal with the infinite boundary condition to solve this equation is one of the main problems of numerical methods. This equation has an important role in development of the boundary layer theory in fluid mechanics. Also, it has provided many good sources of information about the behavior of incompressible boundary layers [22]. In the current work, the Falkner-Skan problem will be solved which is given by the following formula:

$y^{\prime \prime \prime}(x)+y^{\prime \prime}(x) y^{\prime}(x)+\beta\left[\epsilon^{2}-\left(y^{\prime}(x)\right)^{2}\right]=0$

with the following boundary conditions

$y(0)=0, y^{\prime}(0)=1-\epsilon, y^{\prime}(\infty)=\epsilon$.

where is the pressure gradient parameter and $\epsilon$ is velocity ratio parameter. Also, when the value of the parameter $\beta=0$, then Eqution (1) reduces to the Blasius problem which have been studied recently in [30], when $\beta=1$, Eqution (1) reduces to the Hiemenz flow problem and when $\beta=\frac{1}{2}$, Eqution (1) reduces to the Homann flow problem [31].

\section{THE THREE ITERATIVE METHODS TAM, DJM AND BCM}

In this part, the basic idea of the TAM, DJM and BCM will be introduced.

\subsection{The Basic Concepts of the TAM}

Let us introduce the following nonlinear differential equation [22]:

$$
L(y(x))+N(y(x))+g(x)=0,
$$

with boundary conditions

$$
B\left(y, \frac{d y}{d x}\right)=0
$$


where $x$ is the independent variable, $\mathrm{y}(\mathrm{x})$ is an unknown function $g(x)$ is a known function, $L$ is a linear operator and $N$ is a nonlinear operator and $B$ is the boundary operator. The main condition here in ODE is that $L$ is the linear part. Possible take some linear parts and add them to $N$ as needed.

We first begin by assuming that $y_{0}(x)$ is an initial guess to solve the problem $\mathrm{y}(\mathrm{x})$ and is the solution of the equation

$L\left(y_{0}(x)\right)+g(x)=0, \quad B\left(y_{0}, \frac{d y_{0}}{d x}\right)=0$.

The other approximate solution by solving the next problem

$L\left(y_{1}(x)\right)+g(x)+N\left(y_{0}(x)\right)=0, \quad B\left(y_{1}, \frac{d y_{1}}{d x}\right)=0$.

Thus, we have the following iterative procedure, which is the solution of a linear set of problems

$L\left(y_{n+1}(x)\right)+g(x)+N\left(y_{n}(x)\right)=0, \quad B\left(y_{n+1}, \frac{d y_{n+1}}{d x}\right)=0$

It is important to note that each $y_{i}(x)$ are solutions of the Eqution (2). We take more iterations to solve the problem.

\subsection{Solution of Falkner-Skan problem by the TAM}

We will apply TAM to solve the Falkner-Skan problem given in Eqution (1) with the following boundary conditions:

$y(0)=0, y^{\prime}(0)=1-\epsilon, y^{\prime}(\infty)=\epsilon$.

Applying the TAM, by first distributing the problem as follows:

$L(y)=y^{\prime \prime \prime}(x), N(y)=y^{\prime \prime}(x) y^{\prime}(x)+\beta\left(y^{\prime}(x)\right)^{2}, g(x)=-\beta \epsilon^{2}$.

According with the steps previously presented, we begin to solve the equation

$y_{0}^{\prime \prime \prime}(x)=-\beta \epsilon^{2}, y_{0}(0)=0, y_{0}^{\prime}(0)=1-\epsilon, y_{0}^{\prime \prime}(0)=a$.

Therefore, the initial problem will be:

$L\left(y_{0}\right)=-\beta \epsilon^{2}, y_{0}(0)=0, y_{0}^{\prime}(0)=1-\epsilon, y_{0}^{\prime \prime}(0)=a$.

The following problems can be obtained by the general relationship

$L\left(y_{n+1}(x)\right)+g(x)+N\left(y_{n}(x)\right)=0, y_{n+1}(0)=0, y_{n+1}^{\prime}(0)=1-\epsilon, y_{n+1}^{\prime \prime}(0)=a$.

Then,

$y_{0}^{\prime \prime \prime}(x)=-\beta \epsilon^{2}$

(11) 
By integration the Eqution (11) from 0 to $x$ three times and using the boundary conditions

$$
\begin{aligned}
& y_{0}(0)=0, y_{0}^{\prime}(0)=1-\epsilon \text { and } y_{0}^{\prime \prime}(0)=a, \text { we get } \\
& y_{0}(x)=x+\frac{a x^{2}}{2}-x \in-\frac{1}{6}+x^{3} \beta \epsilon^{2} .
\end{aligned}
$$

The first iteration can be given as follows

$$
y_{1}^{\prime \prime \prime}(x)=-y_{0}^{\prime \prime}(x) y_{0}^{\prime}(x)-\beta \epsilon^{2}+\beta\left(y_{0}^{\prime}(x)\right)^{2} \text { with } y_{1}(0)=0, y_{1}^{\prime}(0)=1-\epsilon \text { and } y_{1}^{\prime \prime}(0)=a \text {. }
$$

By solving the equation (13), we get

$$
\begin{aligned}
& y_{1}(x)=x+\frac{a x^{2}}{2}-\frac{a x^{4}}{24}-\frac{a^{2} x^{5}}{120}+\frac{x^{3} \beta}{6}+\frac{1}{12} a x^{4} \beta+\frac{1}{60} a^{2} x^{5} \beta-x \epsilon+\frac{1}{24} a x^{4} \epsilon-\frac{1}{3} x^{3} \beta \epsilon-\frac{1}{12} a x^{4} \beta \epsilon \\
& +\frac{1}{60} x^{5} \beta \epsilon^{2}+\frac{1}{180} a x^{6} \beta \epsilon^{2}-\frac{1}{60} x^{5} \beta^{2} \epsilon^{2}-\frac{1}{120} a x^{6} \beta^{2} \epsilon^{2}-\frac{1}{60} x^{5} \beta \epsilon^{3}+\frac{1}{60} x^{5} \beta^{2} \epsilon^{3}-\frac{x^{7} \beta^{2} \epsilon^{4}}{1260}+\frac{1}{840} x^{7} \beta^{3} \epsilon^{4} .
\end{aligned}
$$

We obtain the second iteration $y_{2}(x)$ by solving the following

$$
y_{2}^{\prime \prime \prime}(x)=-y_{1}^{\prime \prime}(x) y_{1}^{\prime}(x)-\beta \epsilon^{2}+\beta\left(y_{1}^{\prime}(x)\right)^{2} \text { with } y_{2}(0)=0, y_{2}^{\prime}(0)=1-\epsilon \text { and } y_{2}^{\prime \prime}(0)=a \text {. }
$$

Then, by solving the Eqution (14), we get:

$$
\begin{aligned}
& y_{2}(x)=x+\frac{a x^{2}}{2}-\frac{a x^{4}}{24}-\frac{a^{2} x^{5}}{120}+\frac{a x^{6}}{240}+\frac{11 a^{2} x^{7}}{5040}+\frac{11 a^{3} x^{8}}{40320}-\frac{a^{2} x^{9}}{24192}-\frac{a^{3} x^{10}}{64800}-\frac{a^{4} x^{11}}{712800}+\frac{x^{3} \beta}{6} \\
& +\frac{1}{12} a x^{4} \beta-\frac{x^{5} \beta}{60}+\frac{1}{60} a^{2} x^{5} \beta-\frac{1}{60} a x^{6} \beta-\frac{2}{315} a^{2} x^{7} \beta+\frac{a x^{8} \beta}{2688}+\ldots+\frac{x^{15} \beta^{5} \epsilon^{8}}{24766560} \\
& -\frac{23 x^{15} \beta^{6} \epsilon^{8}}{412776000}+\frac{x^{15} \beta^{7} \epsilon^{8}}{39312000} .
\end{aligned}
$$

We will continue to get the other approximations till $n=5$, for $y_{n}(x)$ but not all terms are listed, for the sake of brevity.

After finding the approximate solution of the series $\left(y_{5}(x)\right)$ which contains the value of the missing condition $\left(y_{5}^{\prime \prime}(0)=a\right)$, then to find the numerical value of $(a)$, we use the Padé approximation to $y(x)$, which is the division between two polynomials are $\mathrm{Q}_{m}(x)$ and $\mathrm{R}_{n}(x)$ of degrees $\mathrm{m}$ and $\mathrm{n}$ respectively and given by the following relationship[32]. 
$p(x)=\frac{Q_{m}(x)}{R_{n}(x)}=\frac{\sum_{i=0}^{m} a_{i} x^{i}}{1+\sum_{j=0}^{n} b_{j} x^{j}}=\frac{a_{0}+a_{1} x+a_{2} x^{2}+\ldots .+a_{m} x^{m}}{1+b_{0}+b_{1} x+b_{2} x^{2}+\ldots .+b_{n} x^{n}}$.

Now, by applying the Padé approximation for $\left(\mathrm{y}_{5}^{\prime}(x)\right)$ (since the third used boundary condition $\left.y^{\prime}(\infty)=\epsilon\right)$ with $(\beta=0.5, \epsilon=0.1)$, we get [2]

$P_{2}^{2}\left(y^{\prime}{ }_{5}(x)\right)=\frac{0.9+(0.9(0 .-0.375 a)+a) x+(0.2675+(0 .-0.375 a) a) x^{2}}{1+(0 .-0.375 a) x+0.075 x^{2}}$,

By taking $\lim _{x \rightarrow \infty} P_{2}^{2}(x)$, we obtain

$P_{2}^{2}(x)=3.56667-5 a^{2}$,

By applying the condition value $y^{\prime}(\infty)=\epsilon=0.1$, we get

$3.56667-5 a^{2}=0.1$,

Then, the value of $a$ will be $\mathrm{a}= \pm 832666$ this means a dual solution of equation. Thus, we will use the value that achieves better convergence, when $(a=0.832666)$, i.e. . $y^{\prime \prime}(0)=832666$

\subsection{The Basic Concepts of the DJM}

In this part, we consider the nonlinear functional equation as follows [33]:

$$
y=N(y)+f,
$$

where $N$ is a nonlinear operator and $f$ is known function.

Therefore, the solution of Equation (15) takes the following series formula

$$
y=\sum_{i=0}^{\infty} y_{i} .
$$

Now, the nonlinear operator $N$ in Eqution (15) is decomposed as:

$$
N\left(\sum_{i=0}^{\infty} y_{i}\right)=N\left(y_{0}\right)+\sum_{i=0}^{\infty}\left\{N\left(\sum_{j=0}^{i} y_{j}\right)-N\left(\sum_{j=0}^{i-1} y_{j}\right)\right\}
$$

From Equtions. (16) and (17), the Eqution (15) is equivalent to

$$
\sum_{i=0}^{\infty} y_{i}=f+N\left(y_{0}\right)+\sum_{i=0}^{\infty}\left\{N\left(\sum_{j=0}^{i} y_{j}\right)-N\left(\sum_{j=0}^{i-1} y_{j}\right)\right\} \text {. }
$$

Moreover, the recurrence relation can be defined as:

$$
y_{\mathrm{o}}=f \text {, }
$$


$y_{1}=N\left(y_{0}\right)$

$\vdots$

$y_{m+1}=N\left(\sum_{i=0}^{m} y_{i}\right)-N\left(\sum_{i=0}^{m-1} y_{i}\right) \quad m=1,2,3 \ldots$

and

$$
\sum_{i=0}^{m-1} y_{i}=N\left(\sum_{i=0}^{m} y_{i}\right), \quad m=1,2, \ldots .
$$

Then,

$y=f+\sum_{i=0}^{\infty} y_{i}$.

\subsection{Solution The Falkner-Skan Equation by the DJM}

To solve the problem of the Falkner-Skan given in Eqution (1) by the DJM, with boundary conditions:

$y(0)=0, \mathrm{y}^{\prime}(0)=1-\epsilon, \mathrm{y}^{\prime}(\infty)=\epsilon$.

The following steps will be used:

$y^{\prime \prime \prime}(x)=-y(x) y^{\prime \prime}(x)-\beta \epsilon^{2}+\beta\left(y^{\prime}(x)\right)^{2}$.

Integration both sides of Eqution (24) three times from 0 to $x$ with boundary conditions when

$\left(y^{\prime \prime}(0)=a\right)$. According the reducing of multiple integrals [34], the functional Equation(25) become as:

$$
\begin{aligned}
& \mathrm{y}(x)=x+\frac{a x^{2}}{2}-x \epsilon-\frac{1}{6} x^{3} \beta \epsilon^{2}+\int_{0}^{x} \int_{0}^{x} \int_{0}^{x}-y(t) y^{\prime \prime}(t)+\beta\left(y^{\prime}(t)\right)^{2} d t d t d t . \\
& \mathrm{y}(x)=x+\frac{a x^{2}}{2}-x \epsilon-\frac{1}{6} x^{3} \beta \epsilon^{2}+\frac{1}{2} \int_{0}^{x}(x-t)^{2}\left(-y(t) y^{\prime \prime}(t)+\beta\left(y^{\prime}(t)\right)^{2}\right) d t .
\end{aligned}
$$

Then

$$
\begin{aligned}
& y_{0}(x)=x+\frac{a x^{2}}{2}-x \epsilon-\frac{1}{6} x^{3} \beta \epsilon^{2}, \\
& N\left(y_{n+1}\right)=\frac{1}{2} \int_{0}^{x}(x-t)^{2}\left(-y_{n}(t) y_{n}^{\prime \prime}(t)+\beta\left(y_{n}^{\prime}(t)\right)^{2}\right) d t, \quad n=0,1,2, \ldots
\end{aligned}
$$

Applying the DJM, we obtain

$$
\begin{aligned}
& y_{0}(x)=x+\frac{a x^{2}}{2}-x \epsilon-\frac{1}{6} x^{3} \beta \epsilon^{2}, \\
& y_{1}(x)=\frac{a x^{4}}{24}-\frac{a^{2} x^{5}}{120}+\frac{x^{3} \beta}{6}+\frac{1}{12} a x^{4} \beta+\frac{1}{60} a^{2} x^{5} \beta+\frac{1}{24} a x^{4} \epsilon-\frac{1}{3} x^{3} \beta \epsilon-\frac{1}{12} a x^{4} \beta \epsilon+\frac{1}{6} x^{3} \beta \epsilon^{2}
\end{aligned}
$$




$$
\begin{aligned}
& +\frac{1}{60} x^{5} \beta \epsilon^{2}+\frac{1}{180} a x^{6} \beta \epsilon^{2}-\frac{1}{60} x^{5} \beta^{2} \epsilon^{2}-\frac{1}{120} a x^{6} \beta^{2} \epsilon^{2}-\frac{1}{60} x^{5} \beta \epsilon^{3}+\frac{1}{60} x^{5} \beta^{2} \epsilon^{3} \\
& -\frac{x^{7} \beta^{2} \epsilon^{4}}{1260}+\frac{1}{840} x^{7} \beta^{3} \epsilon^{4}, \\
& y_{2}=\frac{a x^{6}}{240}+\frac{11 a^{2} x^{7}}{5040}+\frac{11 a^{3} x^{8}}{40320}-\frac{a^{2} x^{9}}{24192}-\frac{a^{3} x^{10}}{64800}-\frac{a^{4} x^{11}}{712800}-\frac{x^{5} \beta}{60}-\frac{1}{60} a x^{6} \beta-\frac{2}{315} a^{2} x^{7} \beta+\frac{a x^{8} \beta}{2688} \\
& -\frac{a^{3} x^{8} \beta}{1260}+\frac{53 a^{2} x^{9} \beta}{181440}+\ldots+\frac{x^{13} \beta^{6} \epsilon^{7}}{1235520}-\frac{x^{15} \beta^{4} \epsilon^{8}}{103194000}+\frac{x^{15} \beta^{5} \epsilon^{8}}{24766560}-\frac{23 x^{15} \beta^{6} \epsilon^{8}}{412776000}+\frac{x^{15} \beta^{7} \epsilon^{8}}{39312000} .
\end{aligned}
$$

Since, that $y_{n}=\sum_{i=0}^{n} y_{i} \quad n=1,2,3, \ldots$

Continue to find other approximations up to till $n=5$, for $y_{n}(x)$ but are not listed for brevity the terms.

We got the same value of $a$ as in subsection 3.2, because the approximate solution is the same.

\subsection{The Basic Concepts of the BCM}

Let us the nonlinear functional equation [28]:

$y(x)=N(y(x))+f(x)$,

when $\mathrm{y}(x)$ is an unknown function which is the main goal, $N$ is nonlinear operator and $f(x)$ is known function.

Now, we will define some successive approximations as follows:

$y_{0}=f$,

$y_{1}=y_{0}+N\left(y_{0}\right)$,

$y_{2}=y_{0}+N\left(y_{1}\right)$,

$\vdots$

$y_{n}=y_{0}+N\left(y_{n-1}\right), \quad n=1,2, \ldots \quad$.

A solution $y$ of Eqution (27) with boundary conditions given by the following

$y=\lim _{n \rightarrow \infty} y_{n}$. 


\subsection{Solution The Falkner-Skan Equation by the BCM}

In this part, the $\mathrm{BCM}$ will be implemented to solve the Falkner-Skan equation, to get an approximate solution.

Followed similar steps as in subsection 3.4, we get an Eqution (26).

Now,

let $y_{0}(x)=0.9 x+0.5 a x^{2}-0.000833333 x^{3}, \quad N\left(y_{n-1}\right)=\frac{1}{2} \int_{0}^{x}(x-t)^{2}\left(-y_{n-1}(t) y_{n-1}{ }^{\prime \prime}(t)+\beta\left(y_{n-1}{ }^{\prime}(t)\right)^{2}\right) d t, \quad n \in N$

We will applying the BCM, we get:

$$
\begin{aligned}
& y_{1}(x)=x+\frac{a x^{2}}{2}-\frac{a x^{4}}{24}-\frac{a^{2} x^{5}}{120}+\frac{x^{3} \beta}{6}+\frac{1}{12} a x^{4} \beta+\frac{1}{60} a^{2} x^{5} \beta-x \epsilon+\frac{1}{24} a x^{4} \epsilon-\frac{1}{3} x^{3} \beta \epsilon-\frac{1}{12} a x^{4} \beta \epsilon \\
& +\frac{1}{60} x^{5} \beta \epsilon^{2}+\frac{1}{180} a x^{6} \beta \epsilon^{2}-\frac{1}{60} x^{5} \beta^{2} \epsilon^{2}-\frac{1}{120} a x^{6} \beta^{2} \epsilon^{2}-\frac{1}{60} x^{5} \beta \epsilon^{3}+\frac{1}{60} x^{5} \beta^{2} \epsilon^{3}-\frac{x^{7} \beta^{2} \epsilon^{4}}{1260}+\frac{1}{840} x^{7} \beta^{3} \epsilon^{4}, \\
& y_{2}(x)=x+\frac{a x^{2}}{2}-\frac{a x^{4}}{24}-\frac{a^{2} x^{5}}{120}+\frac{a x^{6}}{240}+\frac{11 a^{2} x^{7}}{5040}+\frac{11 a^{3} x^{8}}{40320}-\frac{a^{2} x^{9}}{24192}-\frac{a^{3} x^{10}}{64800}-\frac{a^{4} x^{11}}{712800}+\frac{x^{3} \beta}{6} \\
& +\frac{1}{12} a x^{4} \beta-\frac{x^{5} \beta}{60}+\frac{1}{60} a^{2} x^{5} \beta-\frac{1}{60} a x^{6} \beta-\frac{2}{315} a^{2} x^{7} \beta+\frac{a x^{8} \beta}{2688}+\ldots+\frac{x^{15} \beta^{5} \epsilon^{8}}{24766560}-\frac{23 x^{15} \beta^{6} \epsilon^{8}}{412776000}+\frac{x^{15} \beta^{7} \epsilon^{8}}{39312000} .
\end{aligned}
$$

We continue to get other iterations till $n=5$, for $y_{n}(x)$ but are not all listed for brevity, we got the same value of $a$ as for the previous methods: TAM and DJM, since the approximate solutions are the same.

\section{4 .THE ADOMIAN DECOMPOSITION METHOD (ADM)}

In this section the ADM will be introduced to solve the Falkner-Skan equation in a simpler procedure than the work given in [2].

\subsection{The Basic Concepts of the ADM}

We first consider the nonlinear functional equation given in this part [35]

$$
y=N+f
$$

where $N$ is a nonlinear operator, $f$ is a given function.

The solution $y$ is represented as the infinite sum of series

$$
y=\sum_{n=0}^{\infty} y_{n} .
$$

The nonlinear function $N(y)$ is decomposed as follows:

$$
N(y)=\sum_{n=0}^{\infty} A_{n}
$$

where $A_{n}$ are Adomian polynomials and calculated by the formulae 


$$
A_{n}=\frac{1}{n !} \frac{d^{n}}{d \lambda^{n}}\left[N\left(\sum_{i=0}^{\infty} \lambda^{i} A_{i}\right)\right]_{\lambda=0,} \quad n=1,2,3, \ldots
$$

By substituting Equtions (31) and (32) into Eqution (30), we get

$$
\sum_{n=0}^{\infty} y_{n}+\sum_{n=0}^{\infty} A_{n}+f
$$

Thus, the terms of the ADM chain are created as follows:

$$
\left\{\begin{array}{l}
y_{0}=f \\
y_{n}=A_{n-1}, \quad n=1,2,3, \ldots
\end{array}\right.
$$

\subsection{Solution For Falkner-Skan Equation by the ADM}

The ADM will be applied to get the approximate solution for Falkner-Skan problem given in Eqution (1), with boundary conditions, directly without making changes to the equation formula.

While Bakodah et al. [2] suggested transform the problem (1) into the system of differential equation.

Let use integrate both sides of Eqution (24) three times from 0 to $x$ with the use the given boundary conditions with $y^{\prime \prime}(0)=a$, we get

$$
\mathrm{y}(x)=x+\frac{a x^{2}}{2}-x \epsilon-\frac{1}{6} x^{3} \beta \epsilon^{2}+\int_{0}^{x} \int_{0}^{x} \int_{0}^{x}-A_{n}+\beta\left(B_{n}\right) d t d t d t .
$$

where $A_{n}$ and $B_{n}$ are the Adomian polynomials, that represent the nonlinear terms $y_{1}^{\prime \prime}(t) y_{1}^{\prime}(t)$ and respectively. $\left(y_{1}^{\prime}(t)\right)^{2}$

By reducing of multiple integrals, the Eqution (36) becomes:

$$
\mathrm{y}(x)=x+\frac{a x^{2}}{2}-x \epsilon-\frac{1}{6} x^{3} \beta \epsilon^{2}+\frac{1}{2} \int_{0}^{x}(x-t)^{2}\left(-A_{n}+\beta\left(B_{n}\right) d t .\right.
$$

By applying the ADM, we get

$$
\begin{aligned}
& y_{0}=x+\frac{a x^{2}}{2}-x \epsilon-\frac{1}{6} x^{3} \beta \epsilon^{2} \\
& y_{1}=-\frac{a x^{4}}{24}-\frac{a^{2} x^{5}}{120}+\frac{x^{3} \beta}{6}+\frac{1}{12} a x^{4} \beta+\frac{1}{60} a^{2} x^{5} \beta+\frac{1}{24} a x^{4} \epsilon-\frac{1}{3} x^{3} \beta \epsilon-\frac{1}{12} a x^{4} \beta \epsilon+\frac{1}{6} x^{3} \beta \epsilon^{2} \\
& +\frac{1}{60} x^{5} \beta \epsilon^{2}+\frac{1}{180} a x^{6} \beta \epsilon^{2}-\frac{1}{60} x^{5} \beta^{2} \epsilon^{2}-\frac{1}{120} a x^{6} \beta^{2} \epsilon^{2}-\frac{1}{60} x^{5} \beta \epsilon^{3}+\frac{1}{60} x^{5} \beta^{2} \epsilon^{3}-\frac{x^{7} \beta^{2} \epsilon^{4}}{1260}+\frac{1}{840} x^{7} \beta^{3} \epsilon^{4}, \\
& y_{2}=\frac{a x^{6}}{240}+\frac{11 a^{2} x^{7}}{5040}+\frac{11 a^{3} x^{8}}{40320}-\frac{x^{5} \beta}{60}-\frac{1}{60} a x^{6} \beta-\frac{2}{315} a^{2} x^{7} \beta-\frac{a^{3} x^{8} \beta}{1260}+\frac{x^{5} \beta^{2}}{60}+\frac{1}{72} a x^{6} \beta^{2} \\
& +\frac{1}{252} a^{2} x^{7} \beta^{2}+\frac{a^{3} x^{8} \beta^{2}}{2016}-\frac{1}{120} a x^{6} \epsilon-\frac{11 a^{2} x^{7} \epsilon}{5040}+\frac{1}{20} x^{5} \beta \epsilon+\frac{1}{30} a x^{6} \beta \epsilon
\end{aligned}
$$


$-\frac{1}{36} a x^{6} \beta^{2} \epsilon-\frac{1}{252} a^{2} x^{7} \beta^{2} \epsilon+\ldots+\frac{13 x^{9} \beta^{3} \epsilon^{5}}{30240}-\frac{x^{9} \beta^{4} \epsilon^{5}}{5040}-\frac{x^{11} \beta^{3} \epsilon^{6}}{155925}+\frac{19 x^{11} \beta^{4} \epsilon^{6}}{1247400}-\frac{x^{11} \beta^{5} \epsilon^{6}}{118800}$.

We will continue to obtain other iterations till $n=5$, for $y_{n}(\mathrm{x})$ but all are not listed for brevity, when ( $\beta=0.5, \epsilon=1)$, can be find value of $(\mathrm{a}= \pm 832666)$ in the same way used in previous methods.

\section{CONVERGENCE ANALYSIS OF THE SUGGESTED METHODS}

In this part, we prove the convergence analysis for the suggested methods, for nonlinear ordinary differential equations by Eqution(1) with boundary conditions, let us define new iterations as follows.

$$
\begin{aligned}
& f_{0}=y_{0}(x), \\
& f_{1}=F\left[f_{0}\right], \\
& f_{2}=F\left[f_{0}+f_{1}\right], \\
& \vdots \\
& f_{n+1}=F\left[f_{0}+f_{1}+\ldots+f_{n}\right] .
\end{aligned}
$$

Where $F$ is the operator, which is defined as follows

$$
F\left[f_{k}\right]=S_{k}-\sum_{i=0}^{k-1} f_{i}(x), k=1,2, \ldots
$$

The term $S_{k}$ represents the solution of the following equation

$$
L\left(f_{k}(x)\right)+g(x)+N\left(\sum_{i=0}^{k-1} f_{i}(x)\right)=0 \quad \text { with } B\left(f_{k}, \frac{d f_{k}}{d x}\right)=0 .
$$

So, in this way, we get $y(x)=\lim _{n \rightarrow \infty} y_{n}(x)=\sum_{n=0}^{\infty} f_{n}$. Hence, the solution of the problem represented, we can access it by Equtions (37) and (38) in the resulted series

$y(x)=\sum_{i=0}^{\infty} f_{i}(x)$.

The following theorems provide the main results and basic conditions for convergence for the proposed methods $[36,37,38]$.

Theorem 5. 1. Let $F$ be an operator "which is the same operator defined in Eq. 38" from some Hilbert space $H$ to the same space. The solution in a series formula $y_{n}(x)=\sum_{i=0}^{n} f_{i}(x)$ converges if $\exists 0<m<1$ such that $F\left[f_{0}+f_{1}+\ldots+f_{i+1}\right] \leq m F\left[f_{0}+f_{1}+\ldots+f_{i}\right] \quad\left(\right.$ that $\left.\left\|f_{i+1}\right\| \leq m\left\|f_{i}\right\|\right)$ $\forall i=0,1,2,3, \ldots$

This theory is not only a private case of Banach's Fixed-Point theorem, but it is sufficient condition to study the convergent of the TAM, DJM and BCM .

Proof : See [ 36 ].

Theorem 5.2. Let the series solution $y(x)=\sum_{i=0}^{\infty} f_{i}(x)$ be convergent, this series will represented the exact solution of the current nonlinear equation. 
Proof: See [ 36].

Theorem 5.3. Assume that the series solution $\sum_{i=0}^{\infty} f_{i}(x)$ which is defined in $(40)$ be convergent to the solution $y(x)$. If the finite truncated series $\sum_{i=0}^{n} f_{i}(x)$ has been used as an approximation for the solution of the current equation, then the maximum error $E_{n}(x)$ can be evaluated as

$E_{n}(x) \leq \frac{1}{1-m} m^{k+1} f_{0}$.

(41)

Proof : See [ 36 ].

Theorems 5.1 and 5.2 states that the iterative methods solution for the current nonlinear problem. The obtained approximate solutions by any one of the proposed methods converge to the exact solution under the condition $\exists \mathbf{O}<\boldsymbol{m}<\mathbf{1}$ such that $F\left[f_{0}+f_{1}+\ldots+f_{i+1}\right] \leq m F\left[f_{0}+f_{1}+\ldots+f_{i}\right]$ ( that is $\left.\left\|f_{i+1}\right\| \leq a\left\|f_{i}\right\|\right)$ for each $\mathrm{i}=0,1,2,3, \ldots$. i.e, $\forall \mathrm{i}$, if we define the parameters as follows

$P_{i}= \begin{cases}\frac{\left\|f_{i+1}\right\|}{\left\|f_{i}\right\|}, & \left\|f_{i}\right\| \neq 0 \\ 0, & \left\|f_{i}\right\|=0\end{cases}$

where the $L_{2}$-norm uses in Eqution (42) and in section 5, which is define for the vector $\boldsymbol{x}=\left(x_{1}, \ldots, x_{n}\right)$ by:

$$
\|\boldsymbol{x}\|_{2}=\sqrt{\left|x_{1}\right|^{2}+\ldots+\left|x_{n}\right|^{2}}
$$

Therefore, the infinite series solution obtained $\sum_{i=0}^{\infty} f_{i}(x)$ of the current problem, which convergent to the exact solution $y(x)$, when $\mathrm{O} \leq P_{i}<1, \forall i=0,1,2, \ldots$. . Also, just like in Theorem 5.3. The maximum truncation error can be estimated to be $\left\|y(x)-\sum_{i=0}^{n} f_{i}\right\| \leq \frac{1}{1-P} P^{(n+1)}\left\|f_{0}\right\|$ where $P=\max \left\{P_{i}, i=0,1, \ldots, n\right\}$.

\subsection{The Convergence of the TAM}

In this part, we will prove the convergence of the TAM by solving the Falkner-Skan problem, and the convergence condition will be examined as given in Equtions (37) -(38). We get iterative scheme for Eqution (1) given as follows:

$$
f_{0}(x)=0.9 x+0.416333 x^{2}-0.000833333 x^{3} .
$$

We will applyaing the TAM and the operator $F\left[f_{k}\right]$ as defined in Eqution (38) with the term $S_{k}$ which represents the solution ofs the equation

$$
f_{k}^{\prime \prime \prime}(x)=-\left(\sum_{i=0}^{k-1} f_{i}(x)\right)\left(\sum_{i=0}^{k-1} f_{i}^{\prime \prime}(x)\right)-\beta\left[\epsilon^{2}-\left(\sum_{i=0}^{k-1} f_{i}^{\prime}(x)\right)^{2}\right] \text {, with } f_{k}(0)=0,
$$


$f_{k}{ }^{\prime}(0)=1-\epsilon$ and $f_{k}{ }^{\prime \prime}(0)=0.832666$

By applying the above steps, we can get the following terms:

$f_{1}=0.0675 x^{3}+0.0000375 x^{5}+0.0000057824 x^{6}-4.96031746032 \times 10^{-9} x^{7}$,

$f_{2}=-0.0030375 x^{5}-0.000468375 x^{6}-0.0000341518 x^{7}-9.29315 \times 10^{-7} x^{8}-1.49785$

$\times 10^{-7} x^{9}-9.55129 \times 10^{-9} x^{10}-1.63465 \times 10^{-12} x^{11}-3.28546 \times 10^{-12} x^{12}$

$-2.30893 \times 10^{-13} x^{13}+3.93991 \times 10^{-16} x^{14}-1.57723 \times 10^{-19} x^{15}$.

Now, we see that the obtained solution by the TAM which satisfies the convergent conditions by assessment the $P_{i}$ values for this case, where $\beta=0.5, \epsilon=0.1$ we get

$$
\begin{aligned}
& P_{0}=\frac{\left\|f_{1}\right\|_{2}}{\left\|f_{0}\right\|_{2}}=\frac{\sqrt{\left|f_{1}(0)\right|^{2}+\left|f_{1}(0.1)\right|^{2}+\ldots+\left|f_{1}(1)\right|^{2}}}{\sqrt{\left|f_{0}(0)\right|^{2}+\left|f_{0}(0.1)\right|^{2}+\ldots+\left|f_{0}(1)\right|^{2}}}=0.03937809<1, \\
& P_{1}=\frac{\left\|f_{2}\right\|_{2}}{\left\|f_{1}\right\|_{2}}=\frac{\sqrt{\left|f_{2}(0)\right|^{2}+\left|f_{2}(0.1)\right|^{2}+\ldots+\left|f_{2}(1)\right|^{2}}}{\sqrt{\left|f_{1}(0)\right|^{2}+\left|f_{1}(0.1)\right|^{2}+\ldots+\left|f_{1}(1)\right|^{2}}}=0.0452157<1, \\
& P_{2}=\frac{\left\|f_{3}\right\|_{2}}{\left\|f_{2}\right\|_{2}}=\frac{\sqrt{\left|f_{3}(0)\right|^{2}+\left|f_{3}(0.1)\right|^{2}+\ldots+\left|f_{3}(1)\right|^{2}}}{\sqrt{\left|f_{2}(0)\right|^{2}+\left|f_{2}(0.1)\right|^{2}+\ldots+\left|f_{2}(1)\right|^{2}}}=0.07468906<1, \\
& P_{3}=\frac{\left\|f_{4}\right\|_{2}}{\left\|f_{3}\right\|_{2}}=\frac{\sqrt{\left|f_{4}(0)\right|^{2}+\left|f_{4}(0.1)\right|^{2}+\ldots+\left|f_{4}(1)\right|^{2}}}{\sqrt{\left|f_{3}(0)\right|^{2}+\left|f_{3}(0.1)\right|^{2}+\ldots+\left|f_{3}(1)\right|^{2}}}=0.0772255<1, \\
& P_{4}=\frac{\left\|f_{5}\right\|_{2}}{\left\|f_{4}\right\|_{2}}=\frac{\sqrt{\left|f_{5}(0)\right|^{2}+\left|f_{5}(0.1)\right|^{2}+\ldots+\left|f_{5}(1)\right|^{2}}}{\sqrt{\left|f_{4}(0)\right|^{2}+\left|f_{4}(0.1)\right|^{2}+\ldots+\left|f_{4}(1)\right|^{2}}}=0.0730672<1,
\end{aligned}
$$

where, the $P_{i}$ values for $i \geq 0$ and $0 \leq x \leq 1$ are less than1. So the solution for the TAM is convergent.

Since, the approximate solutions for the all suggested methods are equal, therefore, the values of $P_{i}$ are the same, then DJM and BCM approaches are convergent.

\subsection{The Convergence of the ADM}

In this part, we will demonstrate the convergence procedure of the ADM by solving the Falkner-Skan problem. The iterative scheme for Eqution (1) can be written by the following

$f_{0}(x)=0.9 x+0.416333 x^{2}-0.000833333 x^{3}$,

$f_{1}(x)=0.0675 x^{3}+0.0000375 x^{5}+0.0000057824 x^{6}-4.96032 \times 10^{-9} x^{7}$,

$f_{2}(x)=-0.0030375 x^{5}-0.00046837 x^{6}-0.00000160714 x^{7}-9.29315 \times 10^{-7} x^{8}$

$-9.45399 \times 10^{-8} x^{9}+2.06514 \times 10^{-10} x^{10}-1.12734 \times 10^{-13} x^{11}$. 
The solution obtained $\sum_{i=0}^{2} f_{i}(x)$ satisfies the convergent conditions by assessment the $P_{i}$ values for this case, where $\beta=0.5, \epsilon=0.1$, we obtain

where, the $P_{i}$ values $\forall i \geq 0$ and $0 \leq x \leq 1$ are less than 1, so the ADM is convergent.

$$
\begin{aligned}
& P_{0}=\frac{\left\|f_{1}\right\|_{2}}{\left\|f_{0}\right\|_{2}}=\frac{\sqrt{\left|f_{1}(0)\right|^{2}+\left|f_{1}(0.1)\right|^{2}+\ldots+\left|f_{1}(1)\right|^{2}}}{\sqrt{\left|f_{0}(0)\right|^{2}+\left|f_{0}(0.1)\right|^{2}+\ldots+\left|f_{0}(1)\right|^{2}}}=0.0836778<1, \\
& P_{1}=\frac{\left\|f_{2}\right\|_{2}}{\left\|f_{1}\right\|_{2}}=\frac{\sqrt{\left|f_{2}(0)\right|^{2}+\left|f_{2}(0.1)\right|^{2}+\ldots+\left|f_{2}(1)\right|^{2}}}{\sqrt{\left|f_{1}(0)\right|^{2}+\left|f_{1}(0.1)\right|^{2}+\ldots+\left|f_{1}(1)\right|^{2}}}=0.0333195<1, \\
& P_{2}=\frac{\left\|f_{3}\right\|_{2}}{\left\|f_{2}\right\|_{2}}=\frac{\sqrt{\left|f_{3}(0)\right|^{2}+\left|f_{3}(0.1)\right|^{2}+\ldots+\left|f_{3}(1)\right|^{2}}}{\sqrt{\left|f_{2}(0)\right|^{2}+\left|f_{2}(0.1)\right|^{2}+\ldots+\left|f_{2}(1)\right|^{2}}}=0.0346158<1, \\
& P_{3}=\frac{\left\|f_{4}\right\|_{2}}{\left\|f_{3}\right\|_{2}}=\frac{\sqrt{\left|f_{4}(0)\right|^{2}+\left|f_{4}(0.1)\right|^{2}+\ldots+\left|f_{4}(1)\right|^{2}}}{\sqrt{\left|f_{3}(0)\right|^{2}+\left|f_{3}(0.1)\right|^{2}+\ldots+\left|f_{3}(1)\right|^{2}}}=0.00837697<1, \\
& P_{4}=\frac{\left\|f_{5}\right\|_{2}}{\left\|f_{4}\right\|_{2}}=\frac{\sqrt{\left|f_{5}(0)\right|^{2}+\left|f_{5}(0.1)\right|^{2}+\ldots+\left|f_{5}(1)\right|^{2}}}{\sqrt{\left|f_{4}(0)\right|^{2}+\left|f_{4}(0.1)\right|^{2}+\ldots+\left|f_{4}(1)\right|^{2}}}=0.204828<1,
\end{aligned}
$$

\section{NUMERICAL SIMULATION}

To study the accuracy of the approximate solutions of the Falkner-Skan problem by the TAM, DJM, and $\mathrm{BCM}$, it is clear that the exact solution is unknown. The error remainder for the Falkner-Skan problem we can be calculated by

$$
E R_{n}(x)=y^{\prime \prime \prime}(x)+y(x) y^{\prime \prime}(x)+\beta\left[\epsilon^{2}-\left(y^{\prime}(x)\right)^{2}\right] \text {. }
$$

And the maximal error remainder for the Falkner-Skan equation as follows

$M E R_{n}=\max _{0 \leq x \leq 1}\left|E R_{n}(x)\right|$.

the Figure 1, can be see the analysis of the logarithmic plots of the maximum error reminders of the approximate solutions obtained by the suggested methods for the $M E R_{n}$. As well, by increasing the number of iterations, then the errors will be reducing, this indicates the efficiency of the proposed methods. 


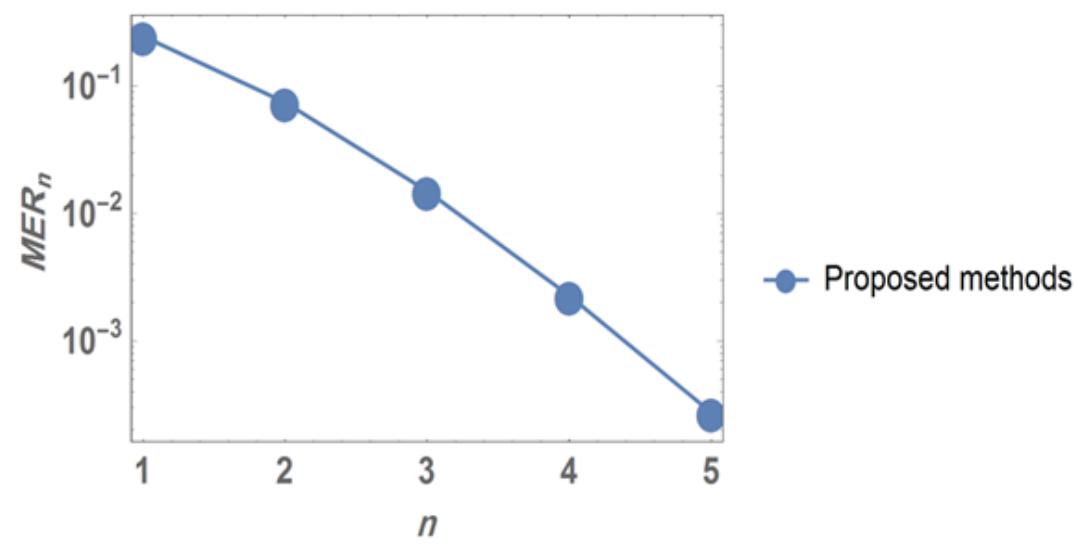

Figure 1. Logarithmic plots for the $M E R_{n}$ versus $n$ from 1 to 5 when $\beta=0.5$ and $\epsilon=0.1$ for FalknerSkan equation by using the proposed methods (TAM, DJM and BCM)

When fixed $\beta=0.5$ and change $\epsilon=0.1,0.2,0.3,0.4,0.5$, we note that the errors will be decreasing and convergence is increasing. When fixed the value of $\epsilon=0.1$ and change the value $\beta=$ $2,3,4,5,6$ the convergence will decrease by the proposed methods.

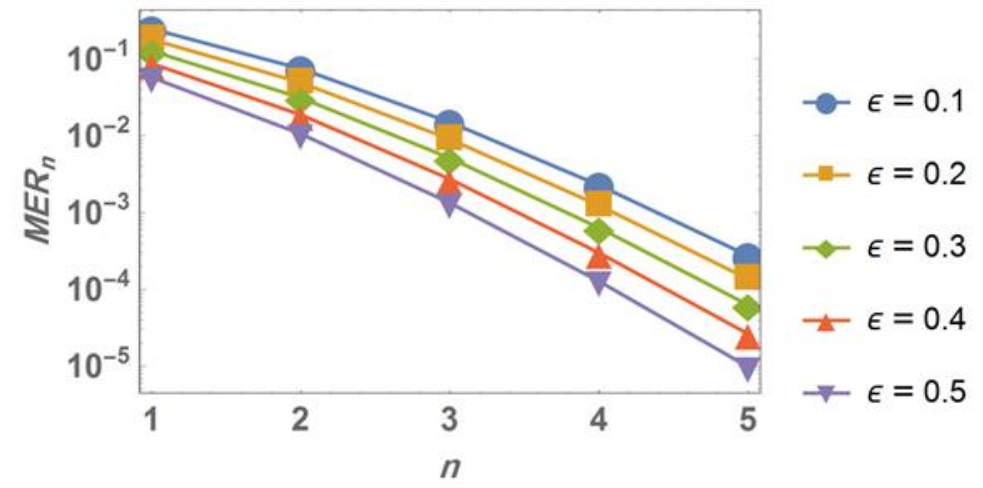

Figure 2. Logarithmic plots for the $M E R_{n}$ versus $n$ from 1 to 5 when $\beta=0.5$ a $\epsilon=0.1,0.2,0.3,0.4,0.5$ for Falkner-Skan equation

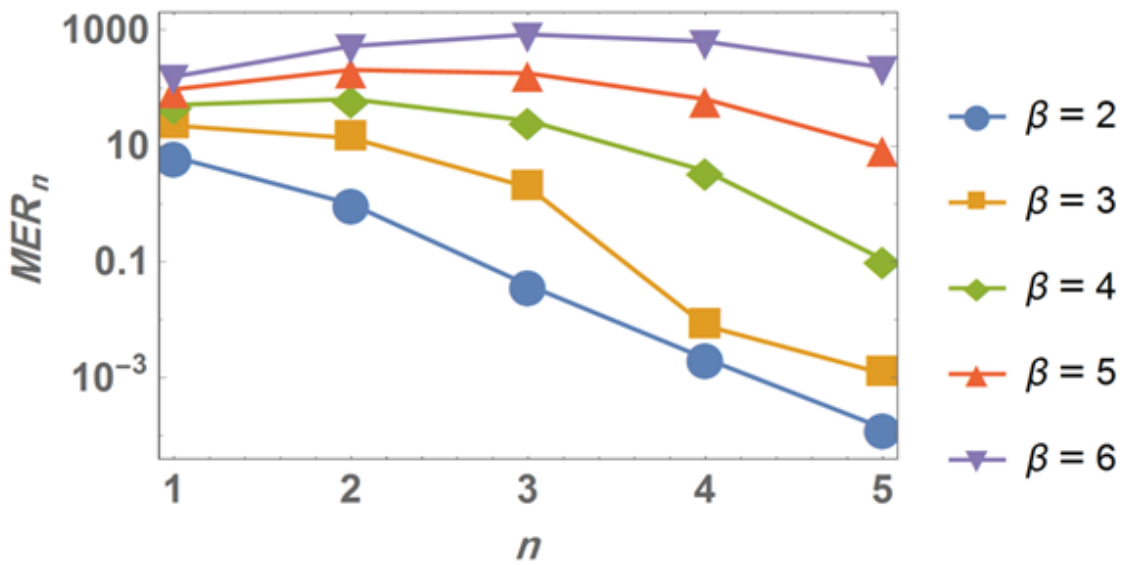

Figure 3. Logarithmic plots for the MER versus $n$ from 1 to 5 when $\epsilon=0.1$ and $\beta=2,3,4,5,6$ for Falkner-Skan problem 
The rate of convergence for the Falkner-skan equation can be estimated by the following formula $\log \left(\frac{M E R_{4}}{M E R_{3}}\right) / \log \left(\frac{M E R_{3}}{M E R_{2}}\right) \simeq 1$.

Moreover, the comparison between approximate solution of the proposed methods with the solution obtained by ADM [23] and VIM [24] is given in Figure 4.

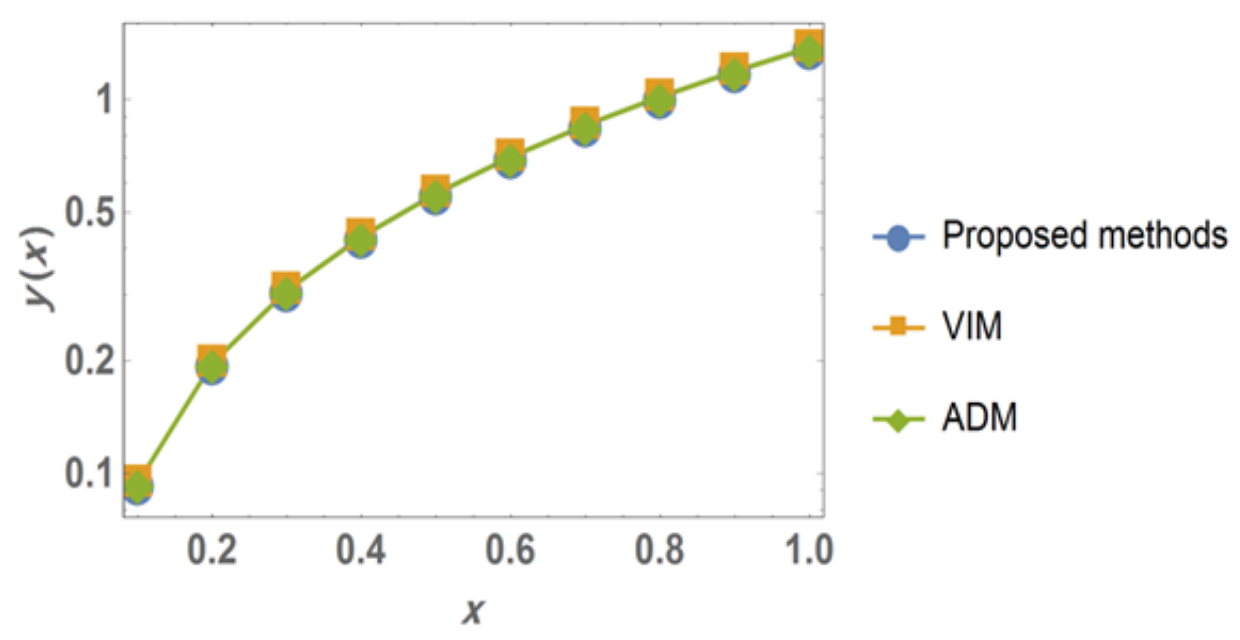

Fiqure 4. Plots for the numerical solution $y(x)$ obtained by each method, versus $x$, when $\epsilon=0.1$ and $\beta=0.5$ for Falkner-Skan equation

Furthermore, the numerical solutions of Falkner-Skan problem by using two numerical methods that are Runge-Kutta 4 (RK4) and Euler (Eul) methods are compare with proposed method is given in Figure 5 and good agreement has been acheived. In Figurs. 6 and 7, we have plotted all the numerical solutions for different values of $\beta$ and $\epsilon$.

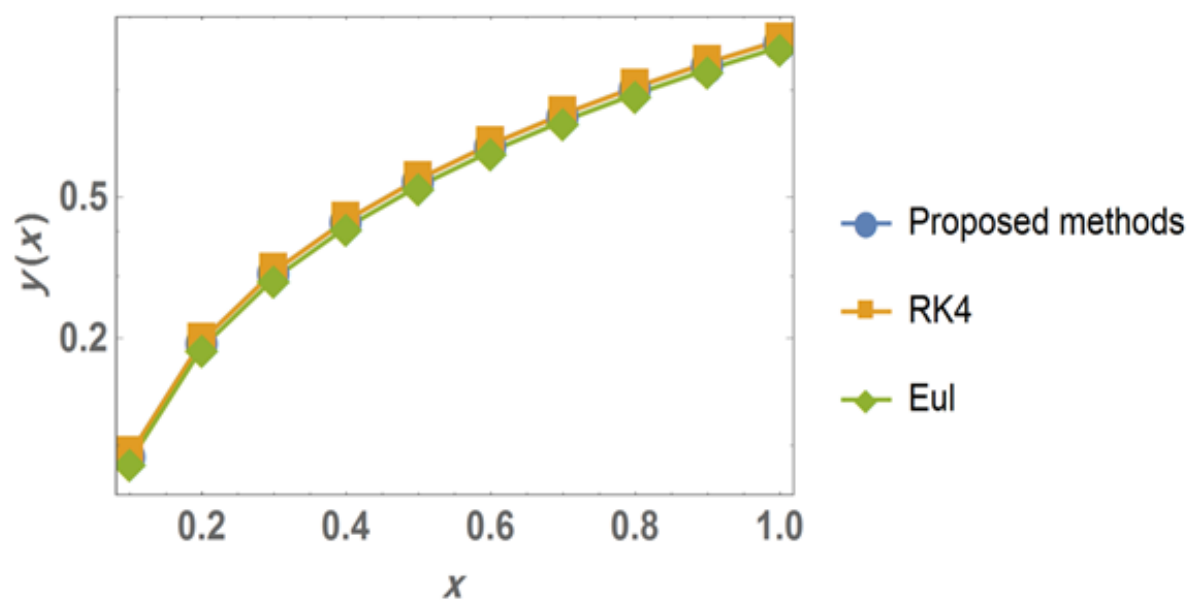

Figure 5. Plots for the numerical solution $y(x)$ obtained by each method, versus $x$, when $\epsilon=0.1$ and $\beta=0.5$ for Falkner-Skan equation 


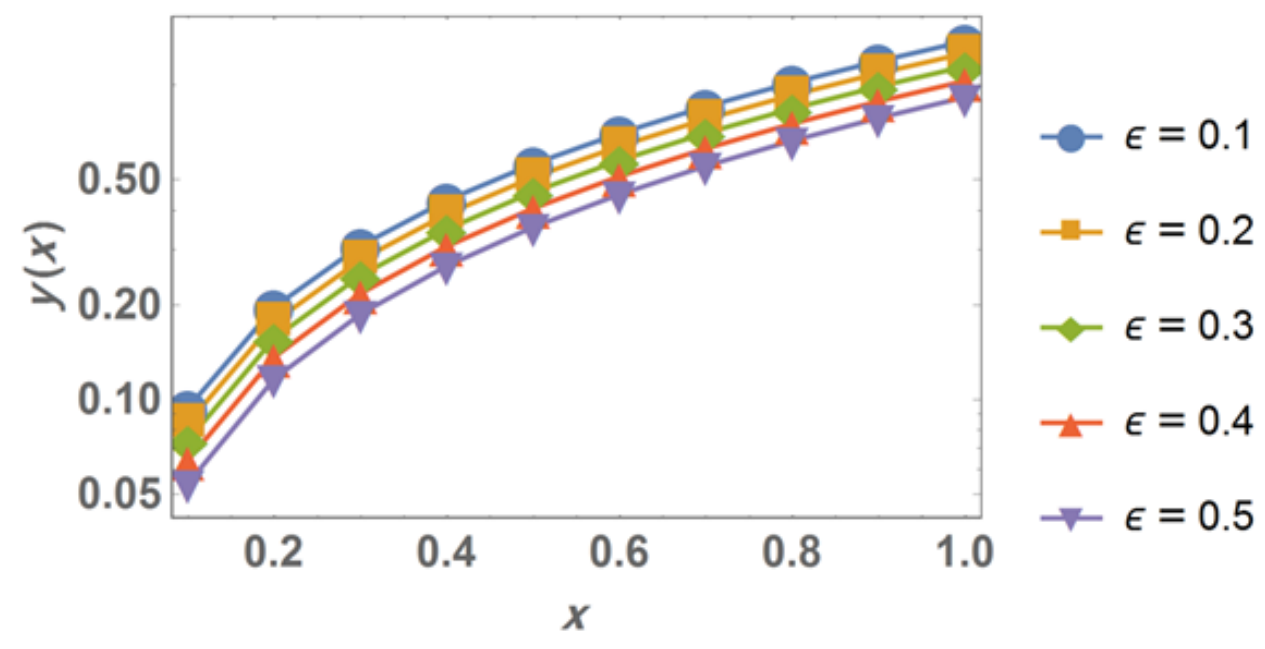

Figure 6. Plots for the numerical solution $y(x)$ obtained by the proposed methods versus $x$, when $\beta=0.5$ and $\epsilon=0.1,0.2,0.3,0.4,0.5$ for Falkner-Skan equation

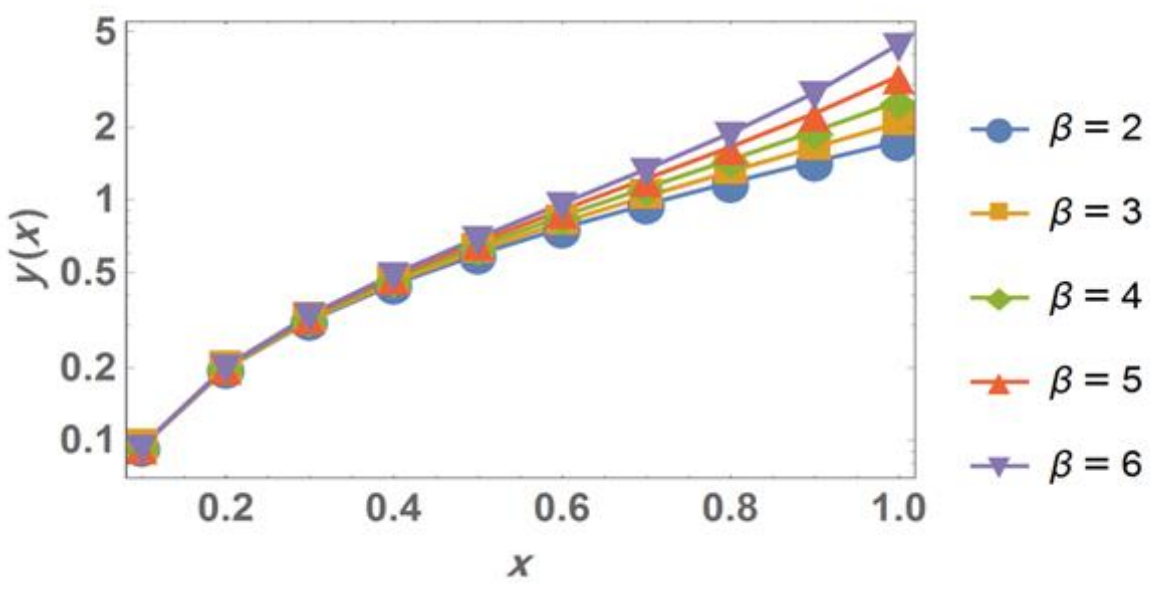

Figure 7. Plots for the numerical solution $y(x)$ obtained by the proposed methods, versus $x$, when $\epsilon=0.1$ and $\beta=2,3,4,5,6$ for Falkner-Skan equation

\section{CONCLUSION}

In this work, we have implemented three iterative methods namely TAM, DJM and BCM to solve FalknerSkan equation. Also, we used another iterative method whichis ADM to solve the problem. We have used Padé approximate method to get the numerical value of the missing boundary conditions. Then we solved the problem by using two numerical methods which are RK 4 and Euler methods, the numerical results of the suggested methods were compared with approximate solutions and good agreement have been achieved.

\section{CONFLICTS OF INTEREST}

No conflict of interest was declared by the authors. 


\section{REFERENCES}

[1] Duque-Daza, C., Lockerby, D., Galeano, C., "Numerical solution of the Falkner-Skan equation using third-order and high-order-compact finite difference schemes", Journal of the Brazilian Society of Mechanical Sciences and Engineering, 33(4): 381-392, (2011).

[2] Bakodah, H., O., Ebaid, A., Wazwaz, A. M., "Analytical and Numerical Treatment Of Falkner-Skan Equation Via A Transformation And Adomian's Method", Romanian Reports in Physics, 70(2): 1-17, (2018).

[3] Dehghan, M., Tatari, M., Azizi, A., "The solution of the Falkner-Skan equation arising in the modelling of boundary-layer problems via variational iteration method", International Journal of Numerical Methods for Heat, Fluid Flow, 21(2): 136-149, (2011).

[4] Eerdun, B., Eerdun, Q. , Huhe, B. , Temuer, C. , Wang, J. Y., "Variational iteration method with He's polynomials for MHD Falkner-Skan flow over permeable wall based on Lie symmetry method", International Journal of Numerical Methods for Heat, Fluid Flow, 24(6): 1348-1362 (2014).

[5] Madaki, A. G., Abdulhameed, M., Ali, M., Roslan, R., "Solution of the Falkner-Skan wedge flow by a revised optimal homotopy asymptotic method", Springer Plus, 5(1): 1-8, (2016).

[6] Temimi, H., Ben-Romdhane, M., "Numerical solution of falkner-skan equation by iterative transformation method", Mathematical Modelling and Analysis, 23(1): 139-151, (2018).

[7] Moallemi, N., Shafieenejad, I., Hashemi, S. F., Fata, A., “Approximate explicit solution of falkner-skan equation by homotopy perturbation method", Engineering and Technology, 4(17): 2893-2897, (2012).

[8] Yao, B., "Approximate analytical solution to the Falkner-Skan wedge flow with the permeable wall of uniform suction", Communications in Nonlinear Science and Numerical Simulation,14(8): 3320-3326, (2009).

[9] Summiya, P., "Numerical solution of the Falkner Skan Equation by using shooting techniques", IOSR Journal of Mathematics, 10( 6): 78-83, (2014).

[10] Kajani, M.T., Maleki, M., Allame, M., "A numerical solution of Falkner-Skan equation via ashifted Chebyshev collocation method”, AIP Conference Proceedings, 1629(1): 381-386, (2014).

[11] Stehlík, P., "On variational methods for periodic discrete problems", Journal of Difference Equations and Applications, 14(3): 259-273, (2008).

[12] Khabir, M. H., Patida, K. C., "Spline approximation method to solve an option pricing problem", Journal of Difference Equations and Applications, 18(11): 1801-1816, (2012) .

[13] Robin, W. A., "Solving differential equations using modified picard iteration", International Journal of Mathematical Education in Science and Technology, 41(5): 649-665, (2010).

[14] Daftardar-Gejji, V., Jafari, H., “An iterative method for solving nonlinear functional equations”, Journal of Mathematical Analysis and Applications, 316(2): 753-763, (2006).

[15] Bhalekar, S., Daftardar-Gejji, V., "New iterative method: Application to partial differential equations", Applied Mathematics and Computation, 203(2): 778-783, (2008). 
[16] Daftardar-Gejji, V., Bhalekar, S., "Solving fractional boundary value problems with Dirichlet boundary conditions using a new iterative method", Computers and Mathematics with Applications, 59(5): 18011809, (2010).

[17] AL-Jawary, M. A., Abd-AL-Razaq, S. G., "Analytic and numerical solution for Duffing equations", International Journal of Basic and Applied Sciences, 5 (2): 115- 119, (2016).

[18] Yaseen, M., Samraiz, M., Naheed, S., “The DJ method for exact solutions of Laplace equation”, Results in Physics, 3: 38- 40, (2013).

[19] Ehsani, F., Hadi, A., Ehsani, F., Mahdavi, R., "An iterative method for solving partial differential equations and solution of Korteweg-de Vries equations for showing the capability of the iterative method", World Applied Programming, 3 (8): 320-327, (2013).

[20] Temimi, H., Ansari, A. R., "A computational iterative method for solving nonlinear ordinary differential equations", LMS Journal of Computation and mathematics, 18 (1): 730-753, (2015).

[21] AL-Jawary, M. A., AL-Qaissy, H. R., "A reliable iterative method for solving Volterra integrodifferential equations and some applications for the Lane-Emden equations of the first kind", Monthly Notices of the Royal Astronomical Society, 448(4): 3093-3104, (2015).

[22] Temimi, H., Ansari, A. R., "A new iterative technique for solving nonlinear second order multi-point boundary value problems", Applied Mathematics and Computation, 218(4): 1457-1466,(2011).

[23] AL-Jawary, M. A., Al-Razaq, S. G., "A semi analytical iterative technique for solving duffing equations", International Journal of pure and applied Mathematics,108(4): 871-885, (2016).

[24] AL-Jawary, M. A. , Raham, R. K., "A semi-analytical iterative technique for solving chemistry problems", Journal of King Saud University, 29 (3): 320-332, (2017).

[25] AL-Jawary, M. A., Hatif, S., "A semi-analytical iterative method for solving differential algebraic equations", Ain Shams Engineering Journal, 9(4): 2581-2586, (2018).

[26] AL-Jawary, M. A., "A semi-analytical iterative method for solving nonlinear thin film flow problems", Chaos. Solitons and Fractals, 99: 52-56, (2017).

[27] AL-Jawary, M. A., Radhi, G., H., Ravnik, J., “A semi-analytical method for solving Fokker-Planck's equations", Journal of the Association of Arab Universities for Basic and Applied Sciences, 24(1): 254262 , (2017).

[28] Daftardar-Gejji, V., Bhalekar, S., "Solving nonlinear functional equation using Banach contraction principle", Far East Journal of Applied Mathematics, 34(3): 303-314, (2009).

[29] Latif, A., "Banach contraction principle and its generalizations", Topics in Fixed Point Theory, 33-64, (2014).

[30] Ebaid, A., Aljoufi, M. D., Wazwaz, A. M., "An advanced study on the solution of nanofluid flow problems via Adomian's method", Applied Mathematics Letters, 46: 117-122, (2015).

[31] Bougoffa, L., Alqahtani, R. T., "Further Solutions Of The Falkner-Skan Equation", Romanian Journal Of Physics, 63:102, (2018). 
[32] Alpaslan, P., H., Oturanc, G., "A Semi Analytical Analysis of a Free Convection Boundary Layer Flow Over a Vertical Plate", arXiv.org,1212.1706, (2012) .

[33] Bhalekar, S., Patade, J., "An Analytical Solution of Fisher's Equation Using Decomposition Method", American Journal of Computational and Applied Mathematics, 6(3):123-127, (2016).

[34] Wazwaz, A. M., “A first course in integral equations”, World Scientific Publishing Company, 328, (2015).

[35] Jiao, Y. C., Dang, C., Yamamoto, Y., “ An extension of the decomposition method for solving nonlinear equations and its convergence", Computers, Mathematics with Applications, 55(4): 760-775, (2008).

[36] Odibat, Z. M., "A study on the convergence of variational iteration method", Mathematical and Computer Modelling, 51(9-10): 1181-1192, (2010).

[37] Monje, Z. A., Ahmed, A.A., "A study of stability of first-order delay differential equations using fixed point theorem banach", Iraqi Journal of Science, 60(12): 2719-2724, (2019).

[38] Abed, S. S., Hasan, Z. M.,"Common fixed point of a finite-step iteration algorithm under total asymptotically quasi-nonexpansive maps”, Baghdad Science Journal, 16(3):654-660, (2019). 